\title{
SPIN-ALLOWED VIBRONIC TRANSITIONS IN MOLECULES: RADIATIVE AND NON-RADIATIVE TRANSITION MOMENTS BY INCLUDING STATIC AND VIBRONIC SPIN-ORBIT COUPLING WITHIN THE ADIABATIC APPROXIMATION
}

\author{
K. GustaV \\ Friedrich-Schiller University Jena, Institute of Physical Chemistry \\ Philosophenweg 14, D(O)-6900 Jena, Federal Republic of Germany \\ (Received June 21, 1991; in final form September 6, 1991)
}

\begin{abstract}
By making use of the second order Herzberg-Teller approach within the adiabatic approximation including the static and vibronic spin-orbit coupling the vibronic transition moments for spin-allowed radiative and non-radiative processes are derived. The role and the contribution of spin-orbit coupling to both transition moments are illustrated.
\end{abstract}

PACS numbers: $33.50 .-\mathrm{j}, 82.50 .-\mathrm{m}$

\section{Introduction}

In a previous paper [1] a refined model for the theoretical treatment of radiative and non-radiative vibronic transition moments was presented for spin-allowed processes which employs the second-order Herzberg-Teller expansion for multi-electron wave functions within the framework of the adiabatic approximation. On that occasion it could be shown that in comparison with the simple model in [2] the refined cut-off criterion yields now essentially modified analytical terms for the vibronic transition moments and thereby also for the corresponding rate constants [1].

Especially, for the internal conversion the theoretical treatment of the vibronic transition moment takes adequately not only the momentum operator but also the kinetic energy operator of vibrational motion into account. Just the latter is not the case when the first-order Herzberg-Teller approach [2] is used. Besides, 
the refined method of treatment [1] makes clear that the customary classification of the active molecular vibrations as promoting and accepting modes has to be modified.

As expected for the refined model [1] in the case of spin-allowed radiative processes, the intensity borrowing in connection with the second-order vibronic coupling is reasonably reflected by the vibronic transition moment. In comparison with the simple treatment [2], in addition, the influence of the "conventional" vibronic coupling in combination with the first derivatives of electronic transition moments of intensity borrowing by all the normal coordinates as well as the intensity borrowing between exclusively virtual excited electronic states initiated through double vibronic couplings are covered for the first time.

On the other hand, from a photophysical viewpoint the refinements achieved for radiative and non-radiative vibronic transition moments, remain naturally still insufficient since both the static spin-orbit and the vibronic spin-orbit couplings being actually always present are not taken into account and, therefore, the electronic states should have pure spin properties.

For this reason the aim of the paper is to derive the radiative and non-radiative vibronic transition moments for spin-allowed processes on the basis of second-order Herzberg-Teller expansion by including the static spin-orbit as well as the first-order vibronic spin-orbit coupling in the electronic state wave functions whereas, finally, all analytical terms higher than second order with regard to the normal coordinates are neglected.

Like in the previous paper [1] it should be stressed, however, that sometimes the dissociative character of vibrational potentials possible in some excited electronic states as shown for benzene and for some simple heterocyclic compounds $[3,4]$, can complicate the problem and makes the use of the harmonic approximation insufficient. In such cases to be proved separately, the vibrational eigenvalue problem has, of course, to be modified. But this question does not alter the general derivation of the physical formulae in the present paper.

\section{Extended model on the basis of the Herzberg-Teller approach with spin-orbit coupling}

The subsequent derivation of radiative and non-radiative vibronic transition moments corresponds largely to the general procedure in [1]. In contrast, the expansion coefficients

$$
\begin{aligned}
& c_{m n}^{(m)}=\sum_{\alpha} A_{m n ; \alpha}^{\prime} Q_{\alpha}^{(m)}+0.5 \sum_{\alpha, \beta} A_{m n ; \alpha \beta}^{\prime \prime} Q_{\alpha}^{(m)} Q_{\beta}^{(m)} \\
& +B_{m n}^{0}+\sum_{\gamma} B_{m n ; \gamma}^{\prime} Q_{\gamma}^{(m)}
\end{aligned}
$$

contain now, however, the additional terms $B_{m n}^{0}$ and $B_{m n ; \gamma}^{\prime}$ which are known to describe the static and vibronic spin-orbit coupling elements [5]. Obviously, these terms are most important to the consideration of the photophysically relevant molecular multiplet system. In addition, it is evident that the existing electronic 
states are, indeed, already mixed spin states. Consequently, the electronic wave functions are by no means exact eigenfunctions of the $\boldsymbol{S}^{2}$-operator. On the other hand, it has to be established that due to their low spin-orbit coupling constants numerous compounds like organic molecules possess merely electronic states with small contributions of different spin behaviour and, on no account, such states with a dominant spin mixing. Therefore, in accordance with literature the radiative and non-radiative vibronic transitions to be regarded are treated as mainly spin-allowed processes. A next paper on spin-forbidden vibronic processes is in preparation.

\subsection{Radiative vibronic transition moment $M_{t w ; s v}$ for spin-allowed processes in consideration of spin-orbit coupling}

On the basis of the relationship (1) within the Herzberg-Teller approximation employing

$$
M_{t w ; s v}=\left\langle X_{t w}\left|M_{t s}\right| X_{s v}\right\rangle
$$

for the vibronic transition moment between singlet states with triplet portions and the expansion of the weakly dynamic electronic transition moment

$$
M_{m n}^{0} \approx \bar{M}_{m n}^{0}+\sum_{\alpha}\left(\partial M_{m n}^{0} / \partial Q_{\alpha}^{(m)}\right)_{0} Q_{\alpha}^{(m)}
$$

with respect to the normal coordinates $Q_{\alpha}^{(m)}$ of initial state $m\left(\bar{M}_{m m}^{0}-\right.$ static electronic transition moment), the following expression (4)

$$
\begin{aligned}
& M_{t w ; s v}=\bar{M}_{t s}^{0}\left\langle X_{t w} \mid X_{s v}\right\rangle+\sum_{\alpha} M_{t s ; \alpha}^{0}\left\langle X_{t w}\left|Q_{\alpha}^{(t)}\right| X_{s v}\right\rangle \\
& +\sum_{j} \bar{M}_{t j}^{0}\left\langle X_{t w}\left|c_{j s}^{(s)}\right| X_{s v}\right\rangle+\sum_{j, \alpha} M_{t j ; \alpha}^{0}\left\langle X_{t w}\left|Q_{\alpha}^{(t)} c_{j s}^{(s)}\right| X_{s v}\right\rangle \\
& +\sum_{i}\left\langle X_{t w}\left|c_{t i}^{(t)}\right| X_{s v}\right\rangle \bar{M}_{i s}^{0}+\sum_{i, \alpha}\left\langle X_{t w}\left|c_{t i}^{(t)} Q_{\alpha}^{(t)}\right| X_{s v}\right\rangle M_{i s ; \alpha}^{0} \\
& +\sum_{i, j}\left\langle X_{t w}\left|c_{t i}^{(t)} c_{j s}^{(s)}\right| X_{s v}\right\rangle \bar{M}_{i j}^{0}+\sum_{i, j, \alpha}\left\langle X_{t w}\left|c_{t i}^{(t)} Q_{\alpha}^{(t)} c_{j s}^{(s)}\right| X_{s v}\right\rangle M_{i j ; \alpha}^{0}
\end{aligned}
$$

is obtained which, for the present, corresponds formally to that in [1]. However, by taking account of the spin selection rules for the electronic transition moments relation (4) can be simplified furthermore to

$$
\begin{aligned}
& M_{t w ; s v}=\bar{M}_{t s}^{0}\left\langle X_{t w} \mid X_{s v}\right\rangle+\sum_{\alpha} M_{i s ; \alpha}^{0}\left\langle X_{t w}\left|Q_{\alpha}^{(t)}\right| X_{s v}\right\rangle \\
& +\sum_{j ; j \varepsilon \text { sing. }} \bar{M}_{t j}^{0}\left\langle X_{t w}\left|c_{j s}^{(s)}\right| X_{s v}\right\rangle+\sum_{j, \alpha ; j \varepsilon \text { sing. }} M_{t j ; \alpha}^{0}\left\langle X_{t w}\left|Q_{\alpha}^{(t)} c_{j s}^{(s)}\right| X_{s v}\right\rangle \\
& +\sum_{i ; i \varepsilon \text { sing. }}\left\langle X_{t w}\left|c_{t i}^{(t)}\right| X_{s v}\right\rangle \bar{M}_{i s}^{0}+\sum_{i, \alpha ; i \varepsilon \text { sing. }}\left\langle X_{t w}\left|c_{t i}^{(t)} Q_{\alpha}^{(t)}\right| X_{s v}\right\rangle M_{i s ; \alpha}^{0}
\end{aligned}
$$




$$
\begin{aligned}
& +\sum_{i, j \varepsilon \text { sing. }}\left\langle X_{t w}\left|c_{t i}^{(t)} c_{j s}^{(s)}\right| X_{s v}\right\rangle \bar{M}_{i j}^{0}+\sum_{i, j \varepsilon \text { tripl. }}\left\langle X_{t w}\left|c_{t i}^{(t)} c_{j s}^{(s)}\right| X_{s v}\right\rangle \bar{M}_{i j}^{0} \\
& +\sum_{i, j, \alpha ; i, j \varepsilon \text { sing. }}\left\langle X_{t w}\left|c_{t i}^{(t)} Q_{\alpha}^{(t)} c_{j s}^{(s)}\right| X_{s v}\right\rangle M_{i j ; \alpha}^{0} \\
& +\sum_{i, j, \alpha ; i, j \varepsilon \text { tripl. }}\left\langle X_{t w}\left|c_{t i}^{(t)} Q_{\alpha}^{(t)} c_{j s}^{(s)}\right| X_{s v}\right\rangle M_{i j ; \alpha}^{0}
\end{aligned}
$$

where the different spin multiplets are marked. Substitution of the expansion coefficients $c_{m n}^{(m)}$ in (5) and application of the second-order cut-off criterion for the vibrational Herzberg-Teller factors lead to

$$
\begin{aligned}
& M_{t w ; s v}=\bar{M}_{t s}^{0}\left\langle X_{t w} \mid X_{s v}\right\rangle+\sum_{\alpha} M_{t s ; \alpha}^{0}\left\langle X_{t w}\left|Q_{\alpha}^{(t)}\right| X_{s v}\right\rangle \\
& +\sum_{j, \mu ; j \varepsilon \text { sing. }} \bar{M}_{t j}^{0} A_{j s ; \mu}^{\prime}\left\langle X_{t w}\left|Q_{\mu}^{(s)}\right| X_{s v}\right\rangle \\
& +0.5 \sum_{j, \mu, \nu ; j \varepsilon \text { sing. }} \bar{M}_{t j}^{0} A_{j s ; \mu \nu}^{\prime \prime}\left\langle X_{t w}\left|Q_{\mu}^{(s)} Q_{\nu}^{(s)}\right| X_{s v}\right\rangle \\
& +\sum_{j, \alpha, \mu ; j \varepsilon \text { sing. }} M_{t j ; \alpha}^{0} A_{j s ; \mu}^{\prime}\left\langle X_{t w}\left|Q_{\alpha}^{(t)} Q_{\mu}^{(s)}\right| X_{s v}\right\rangle \\
& +\sum_{i, \kappa ; i \varepsilon \text { sing. }} A_{i i ; \kappa}^{\prime} \bar{M}_{i s}^{0}\left\langle X_{t w}\left|Q_{\kappa}^{(t)}\right| X_{s v}\right\rangle \\
& +0.5 \sum_{i, \kappa, \lambda ; i \varepsilon \text { sing. }} A_{t i ; \kappa \lambda}^{\prime \prime} \bar{M}_{i s}^{0}\left\langle X_{t w}\left|Q_{\kappa}^{(t)} Q_{\lambda}^{(t)}\right| X_{s v}\right\rangle \\
& +\sum_{i, \kappa, \alpha ; i \varepsilon \text { sing. }} A_{t i ; \kappa}^{\prime} M_{i s ; \alpha}^{0}\left\langle X_{t w}\left|Q_{\kappa}^{(t)} Q_{\alpha}^{(t)}\right| X_{s v}\right\rangle \\
& +\sum_{i, j, \kappa, \mu ; i, j \varepsilon \text { sing. }} A_{i ; ; \kappa}^{\prime} \bar{M}_{i j}^{0} A_{j s ; \mu}^{\prime}\left\langle X_{t w}\left|Q_{\kappa}^{(t)} Q_{\mu}^{(s)}\right| X_{s v}\right\rangle \\
& +\sum_{i, j \varepsilon \text { tripl. }} B_{t i}^{0} \bar{M}_{i j}^{0} B_{j s}^{0}\left\langle X_{t w} \mid X_{s v}\right\rangle+\sum_{i, j, \kappa ; i, j \varepsilon \text { tripl. }} B_{t i ; \kappa}^{\prime} \bar{M}_{i j}^{0} B_{j s}^{0}\left\langle X_{t w}\left|Q_{\kappa}^{(t)}\right| X_{s v}\right\rangle \\
& +\sum_{i, j, \mu ; i, j \varepsilon \text { tripl. }} B_{t i}^{0} \bar{M}_{i j}^{0} B_{j s ; \mu}^{\prime}\left\langle X_{t w}\left|Q_{\mu}^{(s)}\right| X_{s v}\right\rangle \\
& +\sum_{i, j, \kappa, \mu ; i, j \in \text { tripl. }} B_{t i ; \kappa}^{\prime} \bar{M}_{i j}^{0} B_{j s ; \mu}^{\prime}\left\langle X_{t w}\left|Q_{\kappa}^{(t)} Q_{\mu}^{(s)}\right| X_{s v}\right\rangle \\
& +\sum_{i, j, \alpha ; i, j \varepsilon \text { tripl. }} B_{t i}^{0} M_{i j ; \alpha}^{0} B_{j s}^{0}\left\langle X_{t w}\left|Q_{\alpha}^{(t)}\right| X_{s v}\right\rangle \\
& +\sum_{i, j, \kappa, \alpha ; i, j \varepsilon \text { tripl. }} B_{t i ; \kappa}^{\prime} M_{i j ; \alpha}^{0} B_{j s}^{0}\left\langle X_{t w}\left|Q_{\kappa}^{(t)} Q_{\alpha}^{(t)}\right| X_{s v}\right\rangle \\
& +\sum_{i, j, \alpha, \mu ; i, j \varepsilon \text { tripl. }} B_{t i}^{0} M_{i j ; \alpha}^{0} B_{j s ; \mu}^{\prime}\left\langle X_{t w}\left|Q_{\alpha}^{(t)} Q_{\mu}^{(s)}\right| X_{s v}\right\rangle
\end{aligned}
$$

which is rather unwieldy. Due to the large number of terms in (6) and their complexity, especially of the vibrational parts, the use of the noticeable assumption 
$Q_{\alpha}^{(m)}=Q_{\alpha}^{(n)}=Q_{\alpha}$ appears to be necessary, which gives the final expression for the radiative vibronic transition moment $M_{t w ; s v}(t, s-$ spin singlets):

$$
\begin{aligned}
& M_{t w ; s v}=\bar{M}_{t s}^{0}\left\langle X_{t w} \mid X_{s v}\right\rangle+\sum_{\mu}\left[M_{t s ; \mu}^{0}+\sum_{j \varepsilon} \bar{M}_{t j}^{0} A_{j s ; \mu}^{\prime}\right. \\
& \left.+\sum_{i \varepsilon \text { sing. } i \neq t} A_{t i ; \mu}^{\prime} \bar{M}_{i s}^{0}\right]\left\langle X_{t w}\left|Q_{\mu}\right| X_{s v}\right) \\
& +\sum_{\mu, \nu}\left[\sum_{j \varepsilon}\left(0.5 \bar{M}_{t j}^{0} A_{j s ; \mu \nu}^{\prime \prime}+M_{t j ; \mu}^{0} A_{j s ; \nu}^{\prime}\right)\right. \\
& +\sum_{i \varepsilon \text { sing. }, i \neq i}\left(0.5 A_{t i ; \mu \nu}^{\prime \prime} \bar{M}_{i s}^{0}+A_{t i ; \mu}^{\prime} M_{i s ; \nu}^{0}\right) \\
& \left.+\sum_{i, j \varepsilon \text { sing. }, i \neq t, j \neq s} A_{t i ; \mu}^{\prime} \bar{M}_{i j}^{0} A_{j s ; \nu}^{\prime}\right]\left\langle X_{t w}\left|Q_{\mu} Q_{\nu}\right| X_{s v}\right\rangle \\
& +\sum_{i, j \varepsilon \text { tripl. }}\left[B_{t i}^{0} \bar{M}_{i j}^{0} B_{j s}^{0}\left\langle X_{t w} \mid X_{s v}\right\rangle+\sum_{\mu}\left(B_{t i ; \mu}^{\prime} \bar{M}_{i j}^{0} B_{j s}^{0}+B_{t i}^{0} \bar{M}_{i j}^{0} B_{j s ; \mu}^{\prime}\right.\right. \\
& \left.+B_{t i}^{0} M_{i j ; \mu}^{0} B_{j s}^{0}\right)\left\langle X_{t w}\left|Q_{\mu}\right| X_{s v}\right\rangle+\sum_{\mu, \nu}\left(B_{t i}^{0} M_{i j ; \mu}^{0} B_{j s ; \nu}^{\prime}\right. \\
& \left.\left.+B_{t i ; \mu}^{\prime} M_{i j ; \nu}^{0} B_{j s}^{0}+B_{t i ; \mu}^{\prime} \bar{M}_{i j}^{0} B_{j s ; \nu}^{\prime}\right)\left\langle X_{t w}\left|Q_{\mu} Q_{\nu}\right| X_{s v}\right\rangle\right] \text {. }
\end{aligned}
$$

Compared to the analogous radiative vibronic transition moment in [1] the derived expression (7) contains 7 additional terms. These terms are exclusively responsible for the intensity borrowing between virtual excited triplet states, which is realized in cooperation with static and vibronic spin-orbit coupling between the initial and final singlet state, respectively, and excited triplet states. The spin-orbit coupling clearly acts as a spin multiplicity switch within the photophysical term system. Altogether it is noteworthy that its contributions to the radiative vibronic transition moment and to the corresponding rate constant have to be taken also into account in the case of spin-allowed processes.

\subsection{Non-radiative vibronic transition moment $V_{t w ; s v}$ for spin-allowed processes in consideration of spin-orbit coupling}

In contrast to the refined model in [1] the extended treatment on the basis of the static and vibronic spin-orbit coupling within the second-order Herzberg-Teller approach requires the full non-radiative transition operator

$$
J_{\mathrm{N}}=T_{\mathrm{N}}+H_{\mathrm{SO}}
$$

with its two parts $\left(T_{\mathrm{N}}\right.$ : kinetic energy operator of nuclei; $\boldsymbol{H}_{\text {So }}$ spin-orbit coupling operator) what is independent of the spin character of the process. Consequently, 
for the spin-allowed internal conversion (IC) the non-radiative vibronic transition moment

$$
V_{t w ; s v}=\tilde{V}_{t w ; s v}+\tilde{\tilde{V}}_{t w ; s v}
$$

consists of two contributions. Using the operators $\boldsymbol{T}_{\mathrm{N}}$ and $\boldsymbol{H}_{\mathrm{SO}}$ from (8), these terms mean

$$
\tilde{V}_{t w ; s v}=-\hbar^{2} \sum_{\alpha}\left[\left\langle X_{t w}\left|V_{t s ; \alpha}^{\prime}\right| \nabla_{\alpha} X_{s v}\right\rangle+0.5\left\langle X_{t w}\left|V_{t s ; \alpha \alpha}^{\prime \prime}\right| X_{s v}\right\rangle\right]
$$

and

$$
\tilde{\tilde{V}}_{t w ; s v}=-\hbar^{2} \sum_{\alpha}\left[\left\langle X_{t w}\left|B_{t s}\right| X_{s v}\right\rangle+\left\langle X_{t w}\left|\tilde{B}_{t s ; \alpha}^{\prime}\right| Q_{\alpha} X_{s v}\right\rangle\right]
$$

within the basis set of dynamic electronic state wave functions. The following basis transformation into static electronic wave functions necessary within the Herzberg-Teller approach, is carried out by means of the expansion coefficients (1). Because of the complexity of the transformed expressions only terms up to the second order will be covered and, in addition, the assumption $Q_{\alpha}^{(m)}=Q_{\alpha}^{(n)}=Q_{\alpha}$ should be valid like in the case of the radiative vibronic transition moment.

2.2.1. $T_{\mathrm{N}}-p a r t \tilde{V}_{t w ; s v}$

$$
\begin{aligned}
& \tilde{V}_{t w ; s v}=-\hbar^{2}\left[\sum_{\mu} A_{t s ; \mu}^{\prime}\left\langle X_{t w}\left|\partial / \partial Q_{\mu}\right| X_{s v}\right\rangle+\sum_{\mu} A_{t s ; \mu \mu}^{\prime \prime}\left\langle X_{t w}\left|Q_{\mu} \partial / \partial Q_{\mu}\right| X_{t s}\right\rangle\right. \\
& +0.5 \sum_{\mu, \nu ; \mu \neq \nu} A_{t s ; \mu \nu}^{\prime \prime}\left\langle X_{t w}\left|Q_{\nu} \partial / \partial Q_{\mu}\right| X_{s v}\right\rangle+\sum_{\mu} B_{t s ; \mu}^{\prime}\left\langle X_{t w}\left|\partial / \partial Q_{\mu}\right| X_{s v}\right\rangle \\
& +\sum_{j, \mu, \nu} A_{t j ; \mu}^{\prime} A_{j s ; \nu}^{\prime}\left\langle X_{t w}\left|Q_{\mu} \partial / \partial Q_{\nu}\right| X_{s v}\right\rangle+\sum_{j, \mu} B_{t j}^{0} A_{j s ; \mu}^{\prime}\left\langle X_{t w}\left|\partial / \partial Q_{\mu}\right| X_{s v}\right\rangle \\
& +\sum_{j, \mu, \nu} B_{t j ; \mu}^{\prime} A_{j s ; \nu}^{\prime}\left\langle X_{t w}\left|Q_{\mu} \partial / \partial Q_{\nu}\right| X_{s v}\right\rangle+\sum_{j, \mu}^{1} B_{t j}^{0} A_{j s ; \mu \mu}^{\prime \prime}\left\langle X_{t w}\left|Q_{\mu} \partial / \partial Q_{\mu}\right| X_{s v}\right\rangle \\
& +0.5 \sum_{j, \mu, \nu ; \mu \neq \nu} B_{t j}^{0} A_{j s ; \mu \nu}^{\prime \prime}\left\langle X_{t w}\left|Q_{\nu} \partial / \partial Q_{\mu}\right| X_{s v}\right\rangle+\sum_{j, \mu, \nu} A_{t j ; \mu}^{\prime} B_{j s ; \nu}^{\prime} \\
& \times\left\langle X_{t w}\left|Q_{\mu} \partial / \partial Q_{\nu}\right| X_{s v}\right\rangle+\sum_{j, \mu} B_{t j}^{0} B_{j s ; \mu}^{\prime}\left\langle X_{t w}\left|\partial / \partial Q_{\mu}\right| X_{s v}\right\rangle+\sum_{j, \mu, \nu} B_{t j ; \mu}^{\prime} B_{j s ; \nu}^{\prime} \\
& \times\left\langle X_{t w}\left|Q_{\mu} \partial / \partial Q_{\nu}\right| X_{s v}\right\rangle+0.5 \sum_{\mu} A_{t s ; \mu \mu}^{\prime \prime}\left\langle X_{t w} \mid X_{s v}\right\rangle+0.5 \sum_{j, \mu, \nu} A_{t j ; \mu}^{\prime} A_{j s ; \nu \nu}^{\prime \prime} \\
& \times\left\langle X_{t w}\left|Q_{\mu}\right| X_{s v}\right\rangle+0.25 \sum_{j, \mu, \nu, \kappa} A_{t j ; \mu \nu}^{\prime \prime} A_{j s ; \kappa \kappa}^{\prime \prime}\left\langle X_{t w}\left|Q_{\mu} Q_{\nu}\right| X_{s v}\right\rangle+0.5 \sum_{j, \mu} B_{t j}^{0} \\
& \left.\times A_{j s ; \mu \mu}^{\prime \prime}\left\langle X_{t w} \mid X_{s v}\right\rangle+0.5 \sum_{j, \mu, \nu} B_{t j ; \mu}^{\prime} A_{j s ; \nu \nu}^{\prime \prime}\left\langle X_{t w}\left|Q_{\mu}\right| X_{s v}\right\rangle\right] .
\end{aligned}
$$

Taking notice of the fact that the wave functions $\dot{\Phi}_{t}^{0}$ and $\Phi_{s}^{0}$ have spin singlet properties some magnitudes and terms, respectively, must vanish:

$$
B_{t s ; \mu}^{\prime}=0, \quad B_{t j}^{0} A_{j s ; \mu}^{\prime}=0, \quad B_{t j ; \mu}^{\prime} A_{j s ; \nu}^{\prime}=0, \quad B_{t j}^{0} A_{j s ; \mu \nu}^{\prime \prime}=0,
$$




$$
B_{i j}^{0} A_{j s ; \mu \mu}^{\prime \prime}=0, \quad A_{i j ; \mu}^{\prime} B_{j s ; \nu}^{\prime}=0, \quad B_{i j ; \mu}^{\prime} A_{j s ; \nu \nu}^{\prime \prime}=0 .
$$

In this way it is possible to simplify the relation (12) to

$$
\begin{aligned}
& \tilde{V}_{t w ; s v}=-\hbar^{2}\left[0.5 \sum_{\mu} A_{t s ; \mu \mu}^{\prime \prime}\left\langle X_{t w} \mid X_{s v}\right\rangle+\sum_{\mu} A_{t s ; \mu}^{\prime}\left\langle X_{t w}\left|\partial / \partial Q_{\mu}\right| X_{s v}\right\rangle\right. \\
& +\sum_{j, \mu ; j \varepsilon \text { tripl. }} B_{t j}^{0} B_{j s ; \mu}^{\prime}\left\langle X_{t w}\left|\partial / \partial Q_{\mu}\right| X_{s v}\right\rangle \\
& +0.5 \sum_{j, \mu, \nu ; j \varepsilon \text { sing. }} A_{t j ; \mu}^{\prime} A_{j s ; \nu \nu}^{\prime \prime}\left\langle X_{t w}\left|Q_{\mu}\right| X_{s v}\right\rangle \\
& +\sum_{\mu} A_{t s ; \mu \mu}^{\prime \prime}\left\langle X_{t w}\left|Q_{\mu} \partial / \partial Q_{\mu}\right| X_{s \nu}\right\rangle+0.5 \sum_{\mu, \nu ; \mu \neq \nu} A_{t s ; \mu \nu}^{\prime \prime}\left\langle X_{t w}\left|Q_{\nu} \partial / \partial Q_{\mu}\right| X_{s v}\right\rangle \\
& +\sum_{j, \mu, \nu ; j \varepsilon \text { sing. }} A_{t j ; \mu}^{\prime} A_{j s ; \nu}^{\prime}\left\langle X_{t w}\left|Q_{\mu} \partial / \partial Q_{\nu}\right| X_{s v}\right\rangle \\
& +\sum_{j, \mu, \nu ; j \varepsilon \text { tripl. }} B_{t j ; \mu}^{\prime} B_{j s ; \nu}^{\prime}\left\langle X_{t w}\left|Q_{\mu} \partial / \partial Q_{\nu}\right| X_{s v}\right\rangle \\
& \left.+0.25 \sum_{j, \mu, \nu, \kappa ; j \varepsilon \text { sing. }} A_{t j ; \mu \nu}^{\prime \prime} A_{j s ; \kappa \kappa}^{\prime \prime}\left\langle X_{t w}\left|Q_{\mu} Q_{\nu}\right| X_{s v}\right\rangle\right] .
\end{aligned}
$$

The expression (13) obtained substantiates the importance of both the static and vibronic spin-orbit coupling to contribute even to the $\boldsymbol{T}_{\mathrm{N}}$-part of the non-radiative vibronic transition moment $V_{t w ; s v}$. These contributions are clearly a consequence of the partial triplet character of the singlet states. In principle, the spin-orbit interaction is realized only by the involvement of excited triplet states. Ignoring these terms in (13) which actually corresponds to a lower quality of the used electronic wave functions, leads exactly to the same analytical expression derived within the refined model [1].

\subsection{2. $H_{\mathrm{SO}-p a r t} \tilde{\tilde{V}}_{t w ; s v}$}

For spin-allowed processes the derivation of this expression makes use of the relations $B_{t s}^{0}=0$ and $B_{t s ; \alpha}^{\prime}=0$ as well which, generally, are not valid for spin-forbidden transitions. On the basis of equation (11) and the basis transformation according to (1) the following result is obtained:

$$
\begin{aligned}
& \tilde{\tilde{V}}_{t w ; s v}=-\hbar^{2}\left[2 \sum_{j \varepsilon \text { tripl. }} B_{t j}^{0} B_{j s}^{0}\left\langle X_{t w} \mid X_{s v}\right\rangle+\sum_{\mu}\left(2 \sum_{j \varepsilon \text { tripl. }} B_{t j}^{0} B_{j s ; \mu}^{\prime}\right.\right. \\
& +2 \sum_{j \varepsilon \text { tripl. }} B_{t j ; \mu}^{\prime} B_{j s}^{0}+\sum_{i, j ; i \varepsilon \text { sing. }, j \varepsilon \text { tripl. }} A_{t i j \mu}^{\prime} B_{i j}^{0} B_{j s}^{0} \\
& \left.+\sum_{i, j ; i \varepsilon} \sum_{\text {tripl }, j \varepsilon \text { sing. }} B_{t i}^{0} B_{i j}^{0} A_{j s ; \mu}^{\prime}\right)\left\langle X_{t w}\left|Q_{\mu}\right| X_{s v}\right\rangle+\sum_{\mu, \nu}\left(2 \sum_{j \varepsilon \text { tripl. }} B_{t j ; \mu}^{\prime} B_{j s ; \nu}^{\prime}\right.
\end{aligned}
$$




$$
\begin{aligned}
& +\sum_{i, j ; i \varepsilon \text { sing. }, j \varepsilon \text { tripl. }} A_{t i ; \mu}^{\prime} B_{i j}^{0} B_{j s ; \nu}^{\prime}+\sum_{i, j ; i \varepsilon \text { tripl, }, j \varepsilon \text { sing. }} B_{t i ; \mu}^{\prime} B_{i j}^{0} A_{j s ; \nu}^{\prime} \\
& +0.5 \sum_{i, j ; i \varepsilon \text { sing. }, j \varepsilon \text { tripl. }} A_{t i ; \mu \nu}^{\prime \prime} B_{i j}^{0} B_{j s}^{0} \\
& +0.5 \sum_{i, j ; i \varepsilon \text { tripl. }, j \varepsilon \text { sing. }} B_{t i}^{0} B_{i j}^{0} A_{j s ; \mu \nu}^{\prime \prime}+\sum_{i, j ; i \varepsilon \text { sing. }, j \varepsilon \text { tripl. }} A_{t i ; \mu}^{\prime} B_{i j ; \nu}^{\prime} B_{j s}^{0} \\
& \left.\left.+\sum_{i, j ; i \varepsilon} B_{\text {tripl. }, j \varepsilon \text { sing. }} B_{i i}^{0} B_{i j ; \mu}^{\prime} A_{j s ; \nu}^{\prime}\right)\left\langle X_{t w}\left|Q_{\mu} Q_{\nu}\right| X_{s v}\right\rangle\right] .
\end{aligned}
$$

As can be seen from (14) including the spin-orbit coupling in the non-radiative transition operator and, at the same time, in the electronic wave functions specifically contributes also to the total vibronic transition moment of the non-radiative spin-allowed internal conversion. In all terms the coupling elements $B_{m n}^{0}$ and $B_{m n ; \alpha}^{\prime}$ occur which indeed act as double spin multiplicity switches in cooperation or in combination with vibronic couplings of different order, respectively. This mechanism elucidates the necessity to cover not only electronic terms with different spin properties but also with all-valence electron configurations in the theoretical treatment. As in the $T_{\mathrm{N}}$-part of $V_{t w ; s v}$ the promoting modes of the $\boldsymbol{H}_{\mathrm{SO}}$-contribution exhibit the usual de-exciting behaviour as well as exciting properties. Besides, combined promoting modes are possible now in this sense.

Finally it can be stated that the extended treatment of radiative and non-radiative vibronic transition moments may be regarded as a reasonable basis of a photophysical model for spin-allowed processes.

\section{Acknowledgement}

The author is much indebted to the Volkswagen-Stiftung for the financial support.

\section{References}

[1] K. Gustav, Acta Phys. Pol. 80, 521 (1991).

[2] K. Gustav, M. Storch, C. Jung, Acta Phys. Pol. A76, 883 (1989).

[3] S. Kato, J. Chem. Phys. 88, 3045 (1988).

[4] A.L. Sobolewski, W. Domcke, Chem. Phys. Lett. 180, 381 (1991).

[5] R.S. Becker, Theory and Interpretation of Fluorescence and Phosphorescence, Wiley-Interscience, 1968; S.P. McGlynn, T. Azumi, M. Kinoshita, Molecular Spectroscopy of the Triplet State, Prentice Hall, 1969; Organic Molecular Photophysics, Ed. J.B. Birks, Vol. 1, Wiley, London 1973. 